\title{
Polarization-induced Charge Distributions at Polytype Interfaces in Semiconductor Nanostructures
}

\author{
Luying $\mathrm{Li}^{1,2}$ \\ 1. Wuhan National Laboratory for Optoelectronics' Huazhong University of Science and Technology, \\ Wuhan, 430074, China. \\ 2. Department of Physics, Arizona State University, Tempe, Arizona 85287, USA
}

Heterocrystalline polytype structures consist of chemically identical but structurally different materials, which act as barriers for electron transport, leading to charge accumulation in certain regions due either to band offsets at the polytype interfaces [1], or spontaneous polarization in the polytype regions with asymmetric structures [2]. Off-axis electron holography enables quantitative characterization of electrostatic fields and charge distributions at nanometer scale [3], which has been successfully applied to determination of hole accumulations in Ge quantum dots embedded in $\mathrm{Si}$ as well as Ge/Si core/shell nanowires [4-5]. In this work, Charge redistributions at homogeneous Zinc-blende (ZB)/Wurtzite (WZ) heterojunctions in ZnSe nanobelts, as well as InAs nanopillars with 'polytype' heterojunctions, have been studied by off-axis electron holography and other electron microscopy techniques.

Homogeneous ZB/WZ heterostructural junctions have been successfully synthesized in ZnSe nanobelts, and polarity continuity is determined through aberration-corrected HAADF imaging. Using off-axis electron holography, the saw-tooth-like phase shift profile across WZ/ZB/WZ junctions is obtained, while the corresponding thickness profile is comparatively constant, as shown in Figure 1. With the exclusion of possible contributions from piezoelectric polarization and band offsets, spontaneous polarization is identified as the predominant factor causing the experimental phase profile. Our results suggest that the polarization-induced charge distribution at ZB/WZ heterostructural junctions could represent a viable alternative path for tailoring charge distribution in semiconductor nanostructures [2].

Polytype heterocrystalline structures within InAs nanopillars are characterized by multiple TEM techniques, as presented in Figure 2. The electric field related to spontaneous polarization within the ZB region is revealed at nanometer scale using off-axis electron holography, and the measured value of spontaneous polarization for WZ-InAs is close to published results. Moreover, the probe-corrected HAADF imaging technique could provide structural information at atomic resolution, through which the atomic configurations at InAs parital dislocation cores associated with Z-shape faulted dipoles are clearly determined [6]. With careful measurements of the displacements between each $\operatorname{In}^{3}+$ and the center of mass of its nearest neighbors of $\mathrm{As}^{3-}$ within the tetrahedra, variations of local spontaneous polarization are determined with atomic resolution (not shown here), which could be attributed to strain induced by slight lattice mismatch at the interfaces of different polytype structures. The strain-induced variations of spontaneous polarization along the interface normal would provide valuable information for tailoring charge distribution in semiconductor nanostructures and for fabrication of future devices. [7]

\section{References:}

[1] D Spirkoska et al, Phys. Rev. B 80 (2009) p. 245325.

[2] L Li et al, Adv. Mater. 24 (2012), p. 1328. 
[3] M McCartney and D Smith, Annu. Rev. Mater. Res. 37 (2007) p. 729.

[4] L Li et al, Appl. Phys. Lett. 94 (2009), p. 232108.

[5] L Li et al, Nano Lett. 11 (2011), p. 493.

[6] L Li et al, Sci. Rep. 3 (2013), p. 3229.

[7] L Li et al, Adv. Mater. 26 (2014), DOI:10.1002/adma.201304021.

[8] The authors acknowledge funding from the National Science Foundation of China, MOE Doctoral Fund, the Fundamental Research Funds for the Central Universities, SRF for ROCS, SEM. Dr. M. R. McCartney, D. J. Smith and the other collaborators are thanked for their great support and contributions to this work.

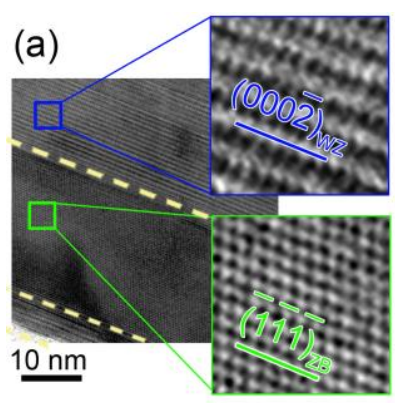

(c)

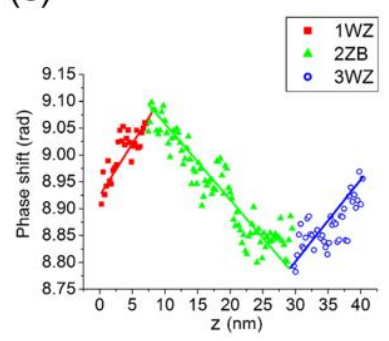

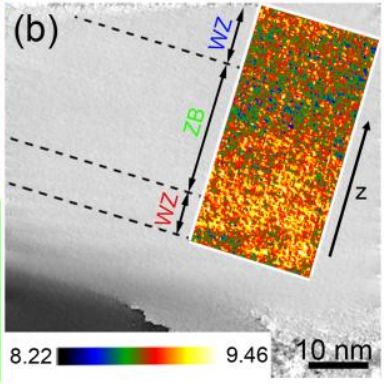

(d)

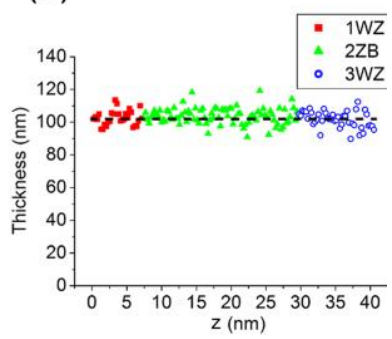

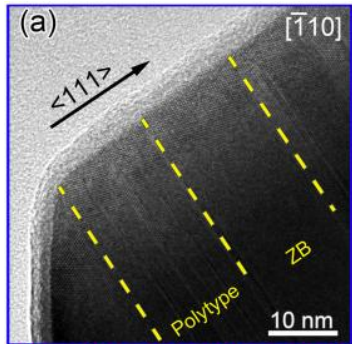

(b)

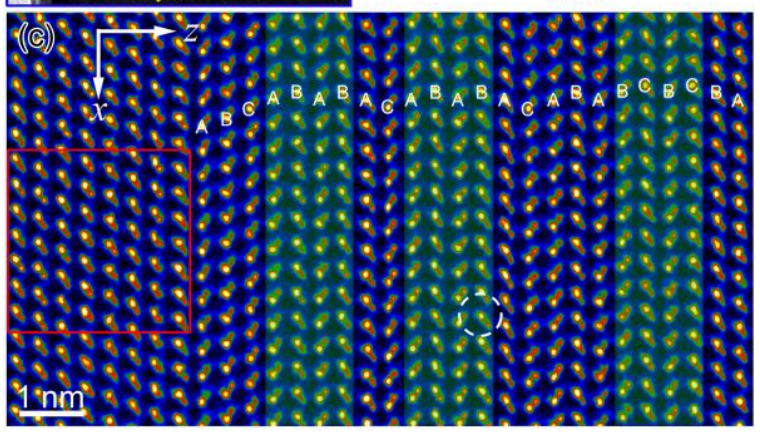

Figure 1 (Left). (a) HREM image of region including WZ/ZB/WZ junctions within ZnSe nanobelt. The projection is along [-110]\|[-2110] $]_{\mathrm{WZ}}$ zone axis. (b) Reconstructed phase image obtained from corresponding off-axis electron hologram: the boxed region used for profiling is shown in color. (c) Phase shift profile of the boxed region labeled in (b). (d) Thickness profile of the same boxed region.

Figure 2 (Right). (a) HREM image of a region including ZB and polytype structures within InAs nanopillar, projected along the [-110] direction. (b) The thickness profile across the polytype/ZB interface (red triangles) is flat across the entire region; the phase shifts in the same region (blue disks) are higher with fluctuations in the polytype region, decreasing linearly in the ZB region. The blue line is a linear fit to phase shifts in the ZB region. (c) Probe-corrected HAADF image of the same InAs nanopillar used for electron holography. Three regions of WZ structure are highlighted by yellow background, from which local displacements between each $\mathrm{In}^{3+}$ and the center of mass of its nearest neighbors of $\mathrm{As}^{3-}$ within the tetrahedral are carefully measured. 